\title{
Pharmacokinetic analysis for the differentiation of pituitary microadenoma subtypes through dynamic contrast-enhanced magnetic resonance imaging
}

\author{
JIAN ZHAI ${ }^{1}$, WENQIANG ZHENG ${ }^{1}$, QIN ZHANG $^{1}$, JIANGFEN WU ${ }^{2}$ and XUEXI ZHANG ${ }^{2}$ \\ ${ }^{1}$ Medical Imaging Centre, The First Affiliated Hospital of Wannan Medical College, Wuhu, Anhui 241000; \\ ${ }^{2}$ GE Healthcare Life Sciences, Shanghai 200000, P.R. China
}

Received April 12, 2018; Accepted December 3, 2018

DOI: $10.3892 / 01.2019 .10083$

\begin{abstract}
The value of pharmacokinetic parameters derived from dynamic contrast-enhanced magnetic resonance imaging (DCE-MRI) in distinguishing pituitary microadenoma subtypes was investigated in the present study. Pathology and follow-up outcomes were applied as the gold standard for differentiating between 76 patients with pituitary microadenomas (38 prolactin-producing tumors, 17 adrenocorticotropic hormone adenomas and 21 growth hormone-producing tumors) and 20 patients with normal pituitary glands. DCE-MRI was conducted to obtain the following quantitative permeability parameters: Volume transfer constant $\left(\mathrm{K}^{\text {trans }}\right)$, rate constant $\left(\mathrm{K}_{\mathrm{ep}}\right)$ and extracellular extravascular volume fraction $\left(\mathrm{V}_{\mathrm{e}}\right)$. Among the 76 cases included, 61 were visually diagnosed using conventional MRI. The $\mathrm{K}^{\text {trans }}, \mathrm{K}_{\mathrm{ep}}$ and $\mathrm{V}_{\mathrm{e}}$ of the microadenoma cases were $0.472 \pm 0.292 / \mathrm{min}, 0.765 \pm 0.359 / \mathrm{min}$ and $0.792 \pm 0.345$, respectively. The $\mathrm{K}^{\text {trans }}, \mathrm{K}_{\mathrm{ep}}$ and $\mathrm{V}_{\mathrm{e}}$ of the normal control group were $0.902 \pm 0.238 / \mathrm{min}, 1.208 \pm 0.599 / \mathrm{min}$ and $0.928 \pm 0.378$, respectively. The $\mathrm{K}^{\text {trans }}$ and $\mathrm{K}_{\mathrm{ep}}$ of patients with microadenomas were significantly lower compared with those of the normal controls $(\mathrm{P}<0.05)$. However, the $\mathrm{V}_{\mathrm{e}}$ of the two groups did not significantly differ. Subtype differentiation analysis revealed that patients with growth hormone-producing tumors exhibited the highest $\mathrm{K}^{\text {trans }}$ value $(\mathrm{P}<0.05) . \mathrm{K}_{\mathrm{ep}}$ significantly differed between growth hormone-producing tumors and the other two subtypes $(\mathrm{P}<0.05)$, but did not significantly differ among three subtypes. Receiver-operator characteristic analysis indicated that the area under the curve values of $\mathrm{K}^{\text {trans }}$ and $\mathrm{K}_{\mathrm{ep}}$ were 0.884 and 0.728 , respectively. Sensitivity and specificity were 95.0 and $82.6 \%$, respectively, when $\mathrm{K}^{\text {trans }}$ was set to $0.614 / \mathrm{min}$ as the cut-off value, and when the $\mathrm{K}_{\mathrm{ep}}$ cut-off value was set to $0.985 / \mathrm{min}$, sensitivity
\end{abstract}

Correspondence to: Professor Jian Zhai, Medical Imaging Centre, The First Affiliated Hospital of Wannan Medical College, 2 West Zheshan Road, Wuhu, Anhui 241000, P.R. China

E-mail: yjszhaij@126.com

Key words: dynamic contrast-enhanced magnetic resonance imaging, pituitary microadenoma, pharmacokinetic parameters and specificity were 60.0 and $81.3 \%$, respectively. In conclusion, $\mathrm{K}^{\text {trans }}$ and $\mathrm{K}_{\mathrm{ep}}$ derived from DCE-MRI could be applied to detect and identify microadenoma subtypes. $\mathrm{K}^{\text {trans }}$ better reflects the blood perfusion alterations exhibited by patients with different microadenoma subtypes.

\section{Introduction}

Pituitary adenomas are one of the most common types of intracranial tumor after meningiomas and gliomas, and they account for $15 \%$ of all intracranial tumor cases (1). The adenomas are also the most common tumors in the sellar regions of the brain and can be classified as macroadenoma or microadenoma on the basis of their size. Microadenomas account for $60-80 \%$ of cases of pituitary adenomas $(2,3)$. Numerous subtypes of microadenomas exist that secrete different hormones, including prolactin, adrenocorticotropic hormones and growth hormones (4). The correct clinical diagnosis of microadenoma and subtype is crucial.

Contrast-enhanced magnetic resonance imaging (CE-MRI) provides richer information on lesion structure and function than conventional MRI. CE-MRI has considerably improved the accuracy of pituitary adenoma diagnosis $(5,6)$. However, the qualitative analysis of CE-MRI images is dependent on scanning devices and protocol parameters. This dependence may weaken repeatability, thus precluding comparisons among multicenter and large-scale studies. Therefore, the recognition and evaluation of pituitary adenomas through dynamic CE-MRI (DCE-MRI) may encounter several difficulties (7).

The quantitative analysis of DCE-MRI images is based on functional MRI and requires the use of specific pharmacokinetic models to evaluate the microvascular density and permeability of tumor lesions $(8,9)$. The present study aimed to diagnose pituitary microadenomas and distinguish microadenoma subtypes on the basis of pharmacokinetic parameters derived from DCE-MRI images. Analysis of variance (ANOVA) tests were employed in the identification of pharmacokinetic parameters. Receiver-operator characteristic (ROC) curves were also used to assess the diagnostic efficiency and optimal cut-off value. The method described in the present study could lead to the development of a clinical microadenoma diagnostic test. 


\section{Materials and methods}

Patients. The present study was approved by the Institutional Review Board of The First Affiliated Hospital of Wannan Medical College (Wuhu, Anhui, China). Written consent was obtained prior to MRI examination. The present study involved 76 patients (58 female and 18 male; age range, $14-61$ years; mean age, $41.3 \pm 12.5$ years) with pituitary microadenomas. Of these 76 cases, 38 patients had prolactin (PRL)-producing tumors, 17 patients had adrenocorticotropic hormone (ACTH) adenoma and 21 patients had somatotropinoma, characterized by excess growth hormone $(\mathrm{GH})$ production. The diagnoses of all the patients were confirmed on the basis of pathology and follow-up outcomes. The diagnoses of 40 patients were confirmed following surgical treatment. The remaining 36 patients were diagnosed on the basis of clinical manifestation and hormone level, as well as through diagnostic treatments and other comprehensive approaches. A total of 20 normal controls (15 female and 5 male; age range, $21-60$ years; mean age, $44.9 \pm 13.3$ years) were also recruited for this study.

All patients with suspected pituitary microadenoma were followed up prior to and following MRI scans. Tumor characteristics and secretion types were determined through a combination of clinical examination and pathological results of therapy or surgery.

MRI examination. A GE Signa HDxt 3.0T MR Scanner with HD8 channel for skull-phased array coils was used in the present study (GE Healthcare Life Sciences, Shanghai, China). Patients were scanned in the supine position. The sagittal view of the sellar region was scanned using the fast spin echo T1-weighted imaging (FSE $\mathrm{T}_{1} \mathrm{WI}$ ) method with the following parameters: Repetition time/echo time/number of excitations (TR/TE/NEX), 4; $40 \mathrm{msec} / 20 \mathrm{msec} / 2$; thickness, $2.5 \mathrm{~mm}$; layer spacing, $0.5 \mathrm{~mm}$; field of view (FOV), 20x20 cm; matrix, 320x224; and echo train length (ETL), 5 . The coronal view of the sellar region was scanned using the FSE $\mathrm{T}_{1}$ WI method with the following parameters: TR/TE/NEX, $500 \mathrm{msec} / 20 \mathrm{msec} / 2$; thickness, $2.0 \mathrm{~mm}$; layer spacing, $0.5 \mathrm{~mm}$; FOV, $22 \times 22 \mathrm{~cm}$; matrix, 320x92; and ETL, 8 . The sellar region was then scanned using the FSE $\mathrm{T}_{2} \mathrm{WI}$ method in coronal view with the following parameters: TR/TE/NEX, 2,000 msec/125 msec/2; thickness, $2.0 \mathrm{~mm}$;

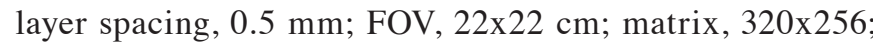
and ETL, 8. A total of 10 scanning slices were obtained. The scanning plane was parallel to the pituitary stalk in the sagittal view and was perpendicular to the major axis of the hypophysis in the coronal view.

For DCE-MRI scanning, Omniscan (gadodiamide; GE Healthcare Life Sciences, Chicago, IL, USA) was intravenously injected using a high-pressure injector. The $\mathrm{T}_{1} \mathrm{WI}$ conventional CE-MRI scan was simultaneously conducted in sagittal and coronal views under the same settings as aforementioned. Conventional CE-MRI was conducted following DCE-MRI scans. DCE-MRI scans were obtained in the coronal view with $\mathrm{T}_{1} \mathrm{WI} 3$-dimensional spoiled gradient recalled acquisition in steady state series. Multi-flip angle protocols with flip angles of 3, 6, 9, 12 and $15^{\circ}$ were first applied for T1 mapping. The DCE-MRI protocol was as follows: TR/TE/NEX,
$5.9 \mathrm{msec} / 1.0 \mathrm{msec} / 1$; thickness, $2.5 \mathrm{~mm}$; layer spacing, $0 \mathrm{~mm}$; FOV, 22x22 cm; matrix, 320x256; and time resolution, $8 \mathrm{sec}$. The central frequency was kept constant through auto pre-scan. The contrast agent $(0.1 \mathrm{mmol} / \mathrm{kg})$ was injected at the rate of $2 \mathrm{ml} / \mathrm{sec}$ into the elbow venous following three phase scans, and then flushed with physiological saline solution $(20 \mathrm{ml})$ at the same rate. The scan was triggered by the injection. Dynamic scanning was performed with the same parameters of the multi-flip angle protocol at $12^{\circ}$. A total of 40 phases were obtained.

Image postprocessing and analysis. DCE-MRI examination was completed by 2 radiology specialists with 14 and 10 years of experience, respectively, using visual observation routine scan, conventional enhanced and dynamic enhanced imaging. Other clinical examination results were used for the independent auxiliary analysis of MR images by the 2 experts, although they were blinded to the clinical symptoms of the patients, experimental standards and other imaging results. Disagreements between the reviewers were resolved through consensus.

All DCE-MRI images were processed by the pharmacokinetics software Omni-Kinetics (version 1; GE Healthcare Life Sciences). Arterial input function (AIF) was placed at the center of the sinus with a size of $\sim 10 \mathrm{~mm}^{2}$. A two-compartment extended Tofts linear model was used to calculate tumor permeability parameters, including volume transfer constant $\left(\mathrm{K}^{\text {trans }}\right)$, rate constant $\left(\mathrm{K}_{\mathrm{ep}}\right)$ and extracellular extravascular volume fraction $\left(\mathrm{V}_{\mathrm{e}}\right)$. The 3 most representative regions of interest of solid lesions with sizes of $20-50 \mathrm{~mm}^{2}$ were selected on MRI images, and the mean value of the 3 regions of interest was exported as the final result.

Statistical analysis. All data were processed and analyzed with SPSS software (version 19.0, IBM Corp., Armonk, NY, USA). Measurements are expressed as the mean \pm standard deviation. An ANOVA was conducted to identify the significance of differences between normal controls and patients with microadenoma. An ANOVA was also conducted to identify differences among microadenoma subtypes. Pairwise comparisons were then performed using the Student-Newman-Keuls algorithm. Enumeration data was analyzed by $\chi^{2}$ test. ROC curves were obtained for the calculation of the area under the curve (AUC), sensitivity and specificity. $\mathrm{P}<0.05$ was considered to indicate a statistically significant difference. The max Youden index was employed to determine the sensitivity, specificity and optimal cut-off points

\section{Results}

Patient population. The present study involved 20 normal controls and 76 patients with pituitary microadenoma (Table I). In general, pituitary tumors were more prevalent among women compared with men. The prevalence of pituitary tumors was not associated with age distribution. The results of $\chi^{2}$ testing demonstrated that the sexes and ages of the patient and control groups were not significantly different. Moreover, secretion types did not significantly differ between the groups. 
Table I. Patient information.

\begin{tabular}{lccrc}
\hline & & \multicolumn{2}{c}{ Sex, $\mathrm{n}$} & \\
\cline { 3 - 4 } Group & Cases, $\mathrm{n}$ & Female & Male & $\begin{array}{c}\text { Age range, } \\
\text { years }\end{array}$ \\
\hline Normal pituitary & 20 & 15 & 5 & $21-60$ \\
Microadenoma & 76 & 58 & 18 & $14-61$ \\
PRL tumor & 38 & 30 & 8 & $25-57$ \\
ACTH tumor & 17 & 12 & 5 & $28-61$ \\
GH tumor & 21 & 16 & 5 & $14-53$ \\
$\chi^{2}$ testing & & $\mathrm{P}>0.05$ & & $\mathrm{P}>0.05$ \\
\hline
\end{tabular}

PRL, prolactin; ACTH, adrenocorticotropic hormone; GH, growth hormone.

Conventional MRI combined with DCE-MRI and image observation. All 76 patients with solitary pituitary microadenomas underwent conventional MRI and DCE-MRI (Fig. 1). Routine MRI easily diagnosed 30 out of 76 patients with pituitary microadenoma, with lesion sizes of $3-10 \mathrm{~mm}$. A total of 17 cases exhibited weak $T_{1}$ WI signals, 10 cases had strong signals and 3 cases showed equal $\mathrm{T}_{1} \mathrm{WI}$ signals. By contrast, 19 cases exhibited equal or strong $\mathrm{T}_{2} \mathrm{WI}$ signals, and the remaining 11 cases had equal or weak signals. Other patients were diagnosed through the comprehensive analysis of MR images.

Comparing the comprehensive evaluations of microadenoma and normal pituitary tissues revealed that the sensitivity and specificity of microadenoma diagnosis were $80.3 \%$ (61/76 patients) and $100 \%$, respectively (Table II).

\section{Quantitative analysis}

Comparison between normal and pituitary microadenomas. Pharmacokinetic parameters derived from DCE-MRI demonstrated that the $\mathrm{K}^{\text {trans }}$ and $\mathrm{K}_{\mathrm{ep}}$ of normal pituitary tissues were significantly higher compared with those of the microadenomas $(\mathrm{P}<0.05)$. However, the normal and disease groups did not exhibit significantly different $\mathrm{V}_{\mathrm{e}}$ (Table III).

Comparison among different pituitary microadenoma subtypes. Comparisons among different pituitary microadenoma subtypes provided different results. The $\mathrm{K}^{\text {trans }}$ values of the GH tumors were significantly higher compared with those of the PRL and ACTH tumors $(\mathrm{P}<0.05)$. The $\mathrm{K}^{\text {trans }}$ values of the ACTH and PRL tumors were not significantly different. The $\mathrm{K}_{\mathrm{ep}}$ values of the GH tumors were higher compared with those of the PRL and ACTH tumors $(\mathrm{P}<0.05)$. The results are provided in Table IV and Fig. 2. However, the $\mathrm{K}_{\mathrm{ep}}$ values of ACTH tumors and PRL tumors were not significantly different. The $\mathrm{V}_{\mathrm{e}}$ values of the three groups did not significantly differ.

Summary of comparisons. Fig. 3 shows a normal pituitary gland. In Fig. 3, the blue color indicates the lowest blood perfusion and permeability, the yellow color indicates a higher perfusion and permeability, and the red indicates the highest blood perfusion and permeability. A relatively higher perfusion and permeability was observed in the gland compared with that in the surrounding issues.

The PRL tumor shown in Fig. 3 was located at the bottom-left of the adenohypophysis. Regions colored blue in the color maps of $\mathrm{K}^{\text {trans }}$ and $\mathrm{K}_{\mathrm{ep}}$ (low value) indicate areas that were affected by microadenoma, and red regions represent normal pituitary tissue on the opposite side (high value). The $\mathrm{V}_{\mathrm{e}}$ map shows mixed colors. The $\mathrm{K}^{\text {trans }}, \mathrm{K}_{\mathrm{ep}}$ and $\mathrm{V}_{\mathrm{e}}$ values of the lesion area were $0.422 / \mathrm{min}, 0.689 / \mathrm{min}$ and 0.741 , respectively.

The ACTH tumor was located in the center of the adenohypophysis. Fig. 3 shows the $\mathrm{K}^{\text {trans }}, \mathrm{K}_{\mathrm{ep}}$ and $\mathrm{V}_{\mathrm{e}}$ of the lesion area. Blue regions in the color maps of $\mathrm{K}^{\text {trans }}, \mathrm{K}_{\mathrm{ep}}$ and $\mathrm{V}_{\mathrm{e}}$ represent areas that were affected by microadenomas. The $\mathrm{K}^{\text {trans }}, \mathrm{K}_{\mathrm{ep}}$ and $\mathrm{V}_{\mathrm{e}}$ values were $0.387 / \mathrm{min}, 0.689 / \mathrm{min}$ and 0.677 , respectively.

The GH tumor was located at the bottom-right of the adenohypophysis. Fig. 3 shows the $\mathrm{K}^{\text {trans }}, \mathrm{K}_{\mathrm{ep}}$ and $\mathrm{V}_{\mathrm{e}}$ of the lesion area. Blue regions in the color maps of $\mathrm{K}^{\text {trans }}, \mathrm{K}_{\mathrm{ep}}$ and $\mathrm{V}_{\mathrm{e}}$ indicate microadenoma-affected areas and provide values of $0.603 / \mathrm{min}, 0.959 / \mathrm{min}$ and 0.906 , respectively.

ROC analysis of quantitative parameters. The ROC curves of $\mathrm{K}^{\text {trans }}$ and $\mathrm{K}_{\mathrm{ep}}$ were drawn in accordance with pathology and follow-up results. The AUC values of $\mathrm{K}^{\text {trans }}$ and $\mathrm{K}_{\mathrm{ep}}$ were 0.884 and 0.728 , respectively. The max Youden index was computed on the basis of the ROC curve to determine the sensitivity, specificity and optimal cut-off points, which are shown in Table V and Fig. 4.

\section{Discussion}

The difficulty in locating a pituitary microadenoma and identifying its subtypes complicates the diagnosis of pituitary microadenomas through clinical examination and conventional MRI. The present results demonstrated that $\mathrm{K}^{\text {trans }}$ and $\mathrm{K}_{\mathrm{ep}}$ calculated from the MRI of patients with pituitary microadenomas were lower compared with those values calculated from the MRI of normal controls. This result contradicts the results obtained for other tumors, including breast, prostate, cervical and esophageal cancer $(10,11)$.

Highly differentiated tumor is associated with high $\mathrm{K}^{\text {trans }}$ and $\mathrm{K}_{\mathrm{ep}}$ values $(12,13)$. Tumor permeability is determined by the levels of vascular endothelium growth factor (VEGF), which increases the number of vascular endothelial cells and promotes angiogenesis and capillary permeability. Furthermore, VEGF mRNA and protein are overexpressed in tumor cells (14). Microvascular density (MVD) could also reflect the number of capillaries in the tumor and is a key factor that influences tumor permeability. Therefore, in certain tumors, the expression level of VEGF and the value of MVD are strongly correlated with the values of $\mathrm{K}^{\text {trans }}$ and $\mathrm{K}_{\mathrm{ep}}(15,16)$.

However, immunohistochemical studies have demonstrated that the oversecretion of certain hormones by pituitary microadenomas decreases VEGF levels relative to those in normal pituitary tissue; this effect may further decrease the MVD value of pituitary microadenomas $(17,18)$. Therefore, the permeability of pituitary microadenomas is lower compared with that of normal pituitary tissue. The present study 
Table II. Diagnosis by conventional MRI.

Gold standard, $\mathrm{n}$

\begin{tabular}{lccc} 
MRI conventional methods & With microadenoma & Without microadenoma & Total, $\mathrm{n}$ \\
\hline With microadenoma & 61 & 0 & 61 \\
Without microadenoma & 15 & 20 & 35 \\
Total & 76 & 20 & 96 \\
\hline
\end{tabular}

MRI, magnetic resonance imaging.

demonstrated that the pathophysiological changes exhibited by pituitary microadenomas contradict those characteristics of other types of tumors.

The $\mathrm{V}_{\mathrm{e}}$ values of patients with pituitary microadenoma were not significantly lower compared with those of the controls. The value of $\mathrm{V}_{\mathrm{e}}$ is inconsistent (19), which may be associated with the presence of edema in tissues that surround pathological regions. $\mathrm{K}_{\mathrm{ep}}$ and $\mathrm{V}_{\mathrm{e}}$ are less effective than $\mathrm{K}^{\text {trans }}$ in distinguishing between healthy and diseased mammary glands (20). In the study, $\mathrm{V}_{\mathrm{e}}$ could not be used to distinguish benign or malignant pituitary tissues. The results may also be affected by the selected AIF (21), which strongly affects the accuracy of the quantitative parameters (22). The vessels of the pathological tissue or the aorta around the pathological tissue must be supplied to reflect the bloodstream in diabetic microangiopathy (23). Therefore, the internal carotid, which surrounds the sellar region, was regarded as the standard AIF and applied in data processing. In addition, the difference in $\mathrm{V}_{\mathrm{e}}$ values may be associated with the different arrival and peak times of the contrast in the vessels. This difference may be attributed to the individual characteristics of the patients.

Radiology specialists have stated that misdiagnosis and overdiagnosis of pituitary microadenoma have become a key problem. The present study demonstrated that the quantitative parameter $\mathrm{K}^{\text {trans }}$ increases the sensitivity of pituitary microadenoma detection, thus increasing diagnostic accuracy. Moreover, determination of the optimal cut-off points also provides quantitative indexes for diagnosis, thus increasing the objectivity of the results. The present study expanded the application of quantitative analysis in the diagnosis of pituitary microadenomas through DCE-MRI. This approach enables comparisons among different niduses and among large-scale and multi-nidus studies.

Previous histopathological studies and radioimmunoassay studies have shown that the level of cyclooxygenase 2 reflects the degree of vascularization (24) and that the level of hematopoietic progenitor cell antigen CD34 (25), an endothelial cell marker, varies in different microadenoma subtypes. The MVD values of ACTH and PRL tumors are lower compared with those of other pituitary microadenoma subtypes and non-functioning adenoma. Moreover, the MVD values of PRL tumors are lower compared with those of pituitary macroadenoma (26). Other types of pituitary microadenoma do not exhibit this characteristic. However, in a systematic study on the degree of vascularization, Jugenburg et al (27) revealed that PRL tumors exhibited the highest MVD values, whereas
Table III. Comparison of quantitative parameters between the patient group and control group.

\begin{tabular}{lcccc}
\hline \multicolumn{5}{c}{ Cases, } \\
Group & $\mathrm{n}$ & $\mathrm{K}^{\text {trans }}, / \mathrm{min}$ & $\mathrm{K}_{\mathrm{ep}}, / \mathrm{min}$ & $\mathrm{V}_{\mathrm{e}}$ \\
\hline Controls & 20 & $0.902 \pm 0.238$ & $1.208 \pm 0.599$ & $0.928 \pm 0.378$ \\
Patients & 76 & $0.472 \pm 0.292$ & $0.765 \pm 0.359$ & $0.792 \pm 0.345$ \\
t & & 6.07 & 3.16 & 1.55 \\
P-value & & $<0.05$ & $<0.05$ & $>0.05$ \\
\hline
\end{tabular}

$\mathrm{K}^{\text {trans }}$, volume transfer constant; $\mathrm{K}_{\mathrm{ep}}$, rate constant; $\mathrm{V}_{\mathrm{e}}$, extracellular extravascular volume fraction.

GH tumors exhibited the lowest MVD values. Nevertheless, the difference between the two values was not statistically significant given the small size of the study sample. ACTH tumors exhibited the highest MVD values and lowest capillary volume, whereas PRL tumors had the highest capillary value (28). These two tumor subtypes have different VEGF levels. Lloyd et al (18) concluded that VEGF expression levels in GH tumors were the highest, those in PRL tumors were the lowest and those in ACTH tumors or non-functioning adenomas were moderate.

Therefore, referring to previous pathology and radioimmunoassay studies, different types of secretory pituitary microadenoma exhibit different pharmacokinetic vascular permeability values. The present study demonstrated that the $\mathrm{K}^{\text {trans }}$ and $\mathrm{K}_{\mathrm{ep}}$ of $\mathrm{GH}$ tumors were the highest, and those of ACTH and PRL tumors were the lowest. The $\mathrm{K}^{\text {trans }}$ and $\mathrm{K}_{\mathrm{ep}}$ of different tumor types, except for those of PRL and ACTH tumors, significantly varied. This result is in accordance with the physiological and pathological variation exhibited by different secretory types (26). These variations may be attributed to the different characteristics of the tumor types. The PRL tumor is the most common tumor, and exhibits slow growth and a small size; it rarely develops into macroadenoma or invades its surrounding tissue. In fact, one-third of patients with PRL tumors tend to experience self-remission (29). ACTH tumors are more invasive than PRL tumors (30), but have lower MVD values and VEGF levels compared with other pituitary microadenoma subtypes. Another factor may cause this behavior. The markers of control cells, including p-27, would decrease the degree of microvascularization (31). Otherwise, dexamethasone inhibits VEGF 
Table IV. Comparison of quantitative parameters between microadenoma subtypes.

\begin{tabular}{lcccc}
\hline Group & Cases, $\mathrm{n}$ & $\mathrm{K}^{\text {trans }}, / \mathrm{min}$ & $\mathrm{K}_{\mathrm{ep}}, / \mathrm{min}$ & $\mathrm{V}_{\mathrm{e}}$ \\
\hline PRL tumor & 38 & $0.428 \pm 0.282$ & $0.675 \pm 0.316$ & $0.772 \pm 0.310$ \\
ACTH tumor & 17 & $0.391 \pm 0.252$ & $0.713 \pm 0.312$ & $0.689 \pm 0.297$ \\
GH tumor & 21 & $0.617 \pm 0.299$ & $0.968 \pm 0.398$ & $0.911 \pm 0.415$ \\
F & & 3.988 & 5.270 & 2.168 \\
P-value & & 0.023 & 0.007 & 0.097 \\
\hline
\end{tabular}

PRL, prolactin; ACTH, adrenocorticotropic hormone; $\mathrm{GH}$, growth hormone; $\mathrm{K}^{\text {trans }}$, volume transfer constant; $\mathrm{K}_{\mathrm{ep}}$, rate constant; $\mathrm{V}_{\mathrm{e}}$, extracellular extravascular volume fraction.

Table V. Receiver-operator characteristic curve of $\mathrm{K}^{\text {trans }}$ and $\mathrm{K}_{\mathrm{ep}}$.

\begin{tabular}{lccc}
\hline Parameter & Area under the curve & Optimal cut point & Sensitivity \\
\hline $\mathrm{K}^{\text {trans }} / \mathrm{min}$ & 0.884 & 0.614 & 0.950 \\
$\mathrm{~K}_{\mathrm{ep}}, \mathrm{min}$ & 0.728 & 0.985 & 0.600 \\
\hline
\end{tabular}

$\mathrm{K}^{\text {trans }}$, volume transfer constant; $\mathrm{K}_{\mathrm{ep}}$, rate constant.

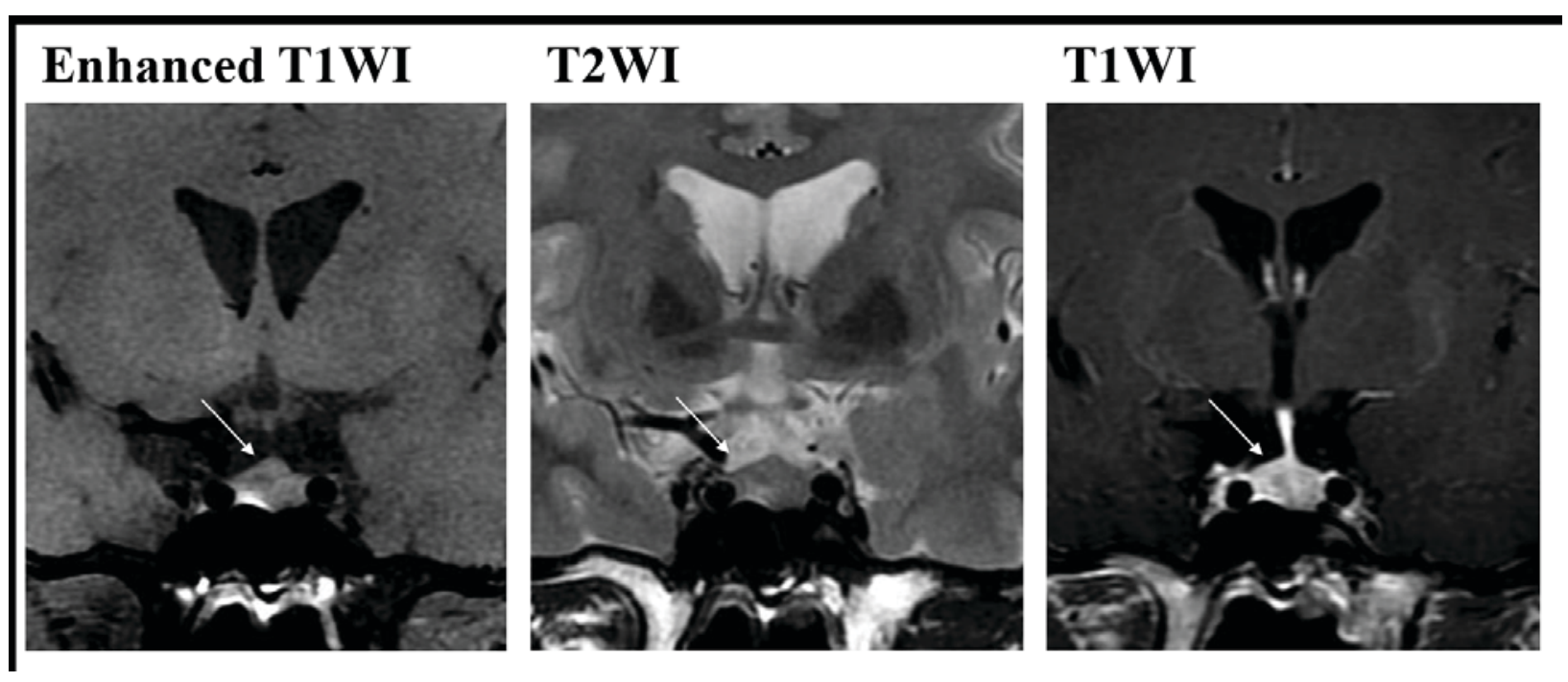

Figure 1. Enhanced $\mathrm{T}_{1} \mathrm{WI}$, and $\mathrm{T}_{2} \mathrm{WI}$ and $\mathrm{T}_{1} \mathrm{WI}$ of pituitary microadenomas at the same position. Nodules exhibit low signal on $\mathrm{T}_{1} \mathrm{WI}$ and marginally high signal on $\mathrm{T}_{2} \mathrm{WI}$ (shown with an arrow). In the enhanced images, the signal intensity of the nidus is weaker compared with that of normal pituitary tissue. $\mathrm{T}_{1} \mathrm{WI}$, T1-weighted image; $\mathrm{T}_{2} \mathrm{WI}, \mathrm{T} 2$-weighted image.

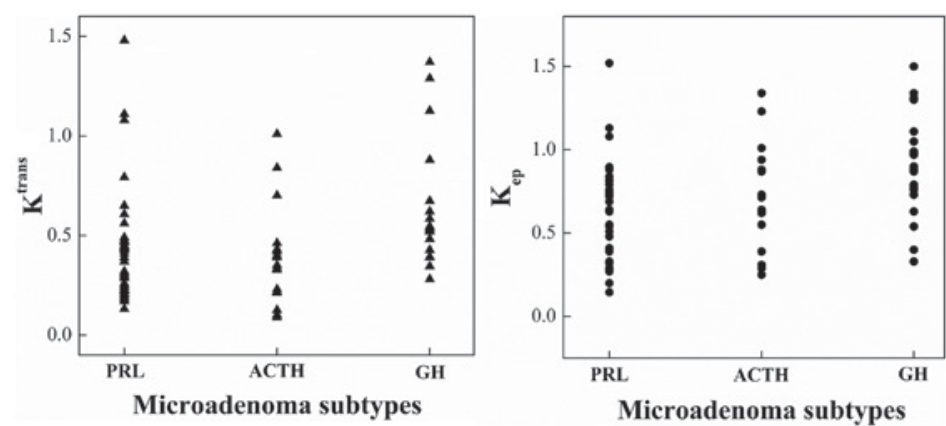

Figure 2. Scatter plots of $K^{\text {trans }}$ and $K_{e p}$ calculated from patients with different pituitary microadenoma subtypes, including PRL, ACTH and GH tumors. The ordinate represents the value of quantitative parameters. $\mathrm{K}^{\text {trans }}$, volume transfer constant; $\mathrm{K}_{\mathrm{ep}}$, rate constant; PRL, prolactin; ACTH, adrenocorticotropic hormone; GH, growth hormone. 

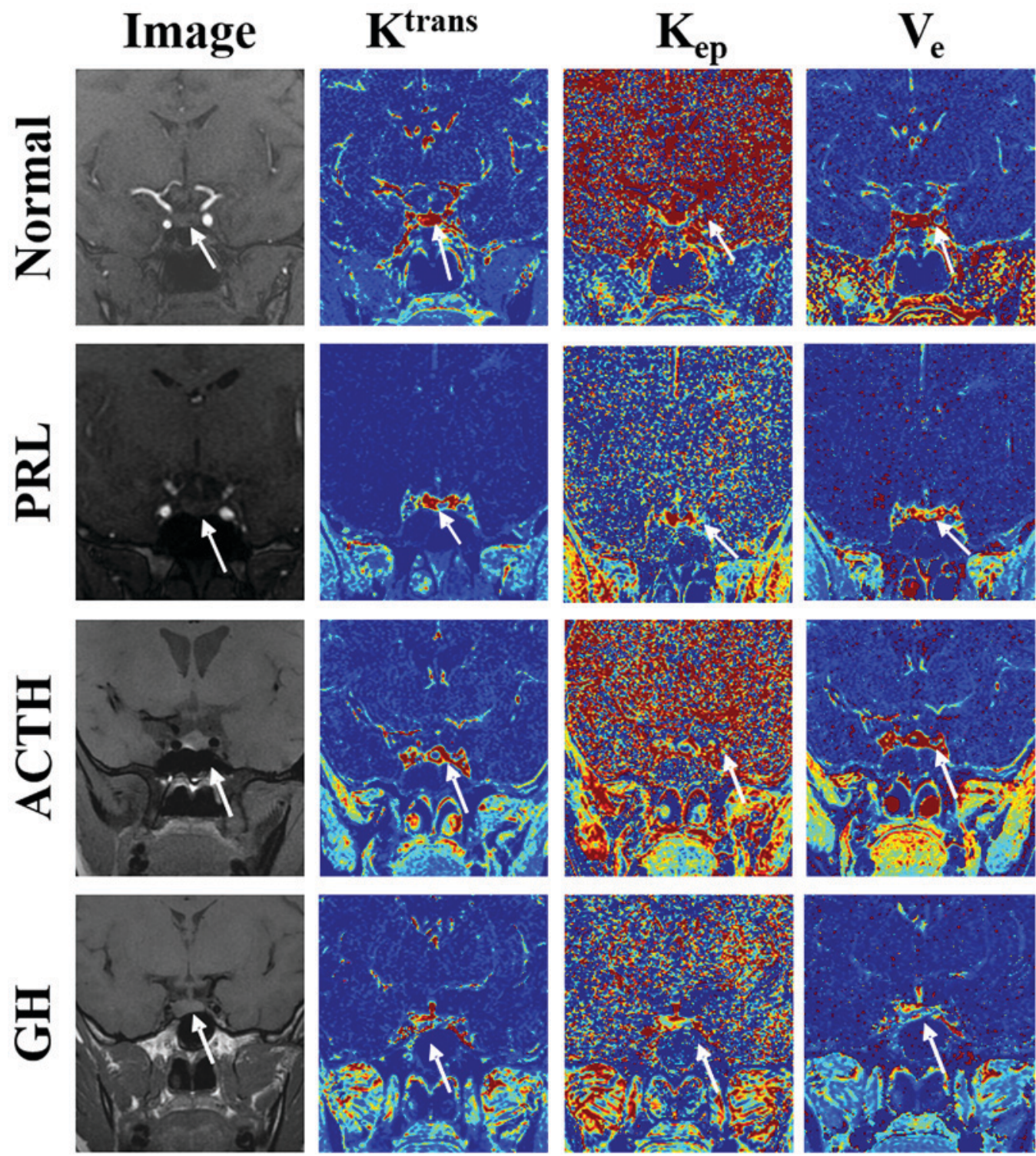

Figure 3. Original setting plans and the color maps of the $K^{\text {trans }}, K_{e p}$, and $V_{e}$ of normal tissue, PRL tumor, ACTH tumor and GH tumor are represented. $K^{\text {trans }}$, volume transfer constant; $\mathrm{K}_{\mathrm{e}}$, rate constant; $\mathrm{V}_{\mathrm{e}}$ extracellular extravascular volume fraction; PRL, prolactin; $\mathrm{ACTH}$, adrenocorticotropic hormone; $\mathrm{GH}$, growth hormone.
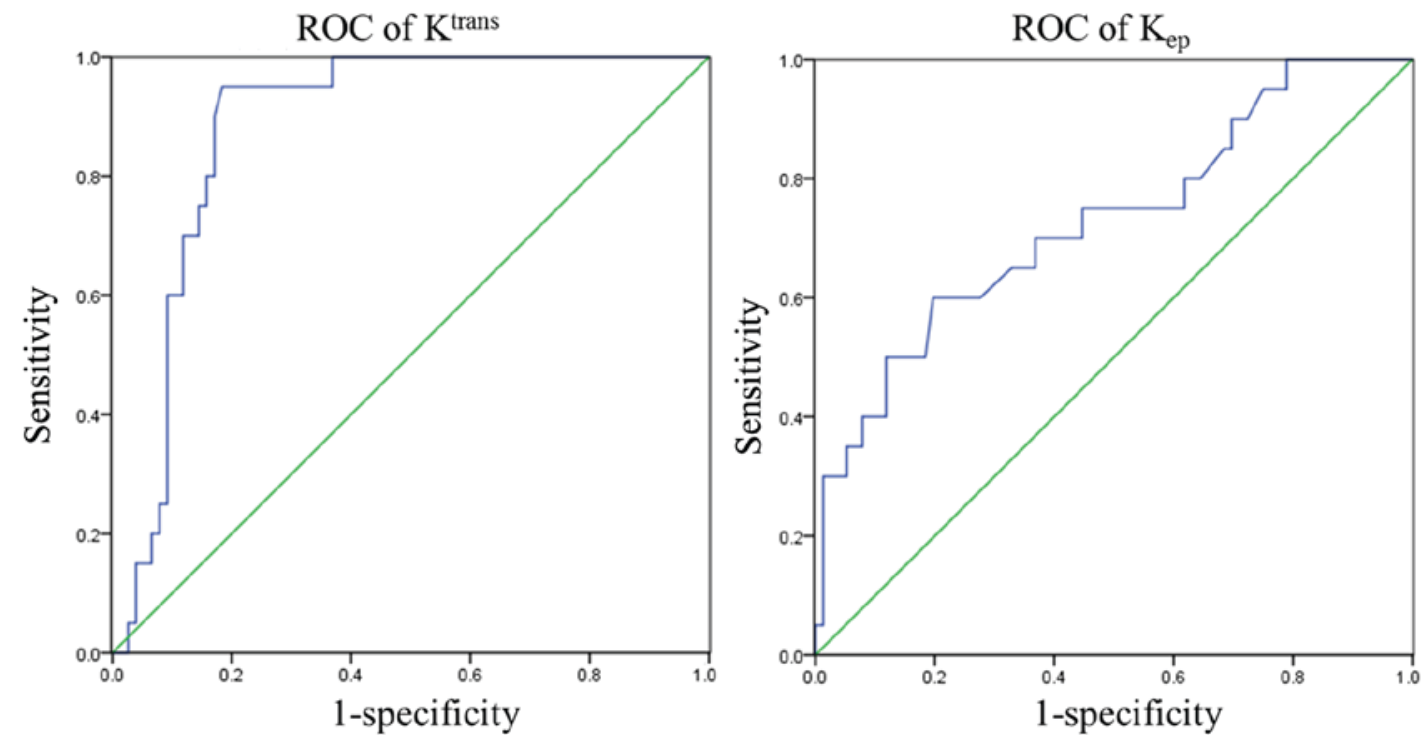

Figure 4. ROC curve of $K^{\text {trans }}$ and $K_{e p}$. ROC, receiver-operator characteristic; $K^{\text {trans }}$, volume transfer constant; $K_{e p}$, rate constant. 
expression in ACTH tumor cells. Therefore, ACTH tumors secrete excess glucocorticoids that would inhibit VEGF expression (32). These factors would decrease microvessel degree and VEGF expression in ACTH tumors relative to those in other pituitary microadenoma subtypes.

The MVD of GH microadenoma is associated with age. Young people are at an increased risk of GH macroadenoma compared with individuals aged $\geq 40$ years $(24)$. The sellar region and its surroundings are easily invaded by adenomas. All the aforementioned factors would increase MVD values and VEGF expression levels. In the present study, the group with GH microadenomas was younger compared with other groups. However, no statistical significance was revealed. Theoretically, the quantitative parameter values of various pituitary microadenoma secretory types may differ and the microvascular permeability of GH tumors is higher compared with that of PRL and ACTH tumors. This phenomenon validates the difference among various pituitary microadenoma secretory types confirmed with histopathology.

The present study demonstrated that quantitative DCE-MRI analysis can be used to classify pituitary microadenomas into different secretory types despite the small sample size. The classification of pituitary microadenomas and the difference in histopathology among various pituitary microadenoma subtypes could be evaluated from pharmacokinetic parameters derived from DCE-MRI. Such an approach could not be achieved through conventional MRI.

The present results indicate that quantitative DCE-MRI analysis could be feasibly applied in the detection of pituitary microadenoma. The quantitative parameters $\mathrm{K}^{\text {trans }}$ and $\mathrm{K}_{\mathrm{ep}}$ could be used to detect and classify pituitary microadenomas. $\mathrm{K}^{\text {trans }}$ could reflect the differences in microcirculation among patients with pituitary microadenoma and its subtypes. The comprehensive evaluation of DCE-MRI is worth adopting over conventional MRI.

At present, the quantitative analysis of DCE-MRI for the diagnosis of pituitary microadenoma remains in the exploration stage. However, the DCE-MRI diagnostic method overcomes the limitations of conventional MRI diagnostic methods and has higher stability and repeatability given that it is independent of machine and scanning parameters. Given the majority of studies have improved protocol settings, optimized postprocessing models and large samples, DCE-MRI could provide highly stable quantitative parameters for the objective diagnosis of pituitary microadenomas. The technique could also be used to examine the difference in pathophysiological changes among various types of secretory tumors. In addition, the quantitative parameters used in the present study could be regarded as biological markers for monitoring the responses of pituitary microadenomas to pharmacotherapy in clinical settings.

\section{Acknowledgements}

Not applicable.

\section{Funding}

No funding was received.

\section{Availability of data and materials}

The data analysis methods used in this study are available from the corresponding author upon reasonable request.

\section{Authors' contributions}

JZ, QZ and WZ designed the study and drafted the manuscript, and were responsible for the MRI scanning. JW and XZ performed the statistical analysis, researched the literature and revised the manuscript. All authors read and approved the final version of the manuscript

\section{Ethics approval and consent to participate}

This study was approved by the Institutional Review Board of The First Affiliated Hospital of Wannan Medical College (Wuhu, Anhui, China). Written consent was provided by all patients.

\section{Patient consent for publication}

Not applicable.

\section{Competing interests}

The authors declare that they have no competing interests.

\section{References}

1. Daly AF, Rixhon M, Adam C, Dempegioti A, Tichomirowa MA and Beckers A: High prevalence of pituitary adenomas: A cross-sectional study in the province of Liege, Belgium. J Clin Endocrinol Metab 91: 4769-4775, 2006.

2. Lieb JM and Ahlhelm FJ: Tumors of the sellar region. Radiologe 57: 740-747, 2017 (In German).

3. Stacey RJ and Powell MP: Sellar and Parasellar Tumors. Neurosurgery: 187-204, 2006.

4. Scheithauer BW, Horvath E, Kovacs K, Laws ER Jr, Randall RV and Ryan N: Plurihormonal pituitary adenomas. Semin Diagn Pathol 3: 69-82, 1986.

5. Shah S, Waldman AD and Mehta A: Advances in pituitary imaging technology and future prospects. Best Pract Res Clin Endocrinol Metab 26: 35-46, 2012.

6. Rand T, Lippitz P, Kink E, Huber H, Schneider B, Imhof H and Trattnig S: Evaluation of pituitary microadenom as with dynamic MR imaging. Eur J Radiol 41: 131-135, 2002.

7. Kopczak A, Renner U and Karl Stalla G: Advances in understanding pituitary tumors. F1000Prime Rep 6: 5, 2014.

8. Murase K: Efficient method for calculating kinetic parameters using T1-weighted dynamic contrast-enhanced magnetic resonance imaging. Magn Reson Med 51: 858-862, 2004.

9. Yankeelov TE, Lepage M, Chakravarthy A, Broome EE, Niermann KJ, Kelley MC, Meszoely I, Mayer IA, Herman CR, McManus K, et al: Integration of quantitative DCE-MRI and ADC mapping to monitor treatment response in human breast cancer: Initial results. Magn Reson Imaging 25: 1-13, 2007.

10. Zhijun Z: Research of DCE-MRI quantitative analysis value in cervical squamous cell carcinomas microvascular permeability. Chin J CT MRI 13: 71-73, 2015 (In Chinese).

11. Lei J, Tian Y,Zhu SC, Han Q, Wei Y, Yang S and Shi DP: Preliminary study of IVIM-DWI and DCE-MRI in early diagnosis of esophageal cancer. Eur Rev Med Pharmacol Sci 19: 3345-3350, 2015.

12. Zhao LY, Zhou CW, Zhang RZ, et al: Correlation of quantitative parameters of dynamic contrast-enhanced MRI with subtypes of breast carcinoma. Chin J Med Imaging Technol 29: 1126-1130, 2013 (In Chinese).

13. Zhao J, Yang ZY, Luo BN, Yang JY and Chu JP: Quantitative evaluation of diffusion and dynamic contrast-enhanced MR in tumor parenchyma and peritumoral area for distinction of brain tumors. PLoS One 10: e0138573, 2015. 
14. Yuan A, Lin CY, Chou CH, Shih CM, Chen CY, Cheng HW, Chen YF, Chen JJ, Chen JH, et al: Functional and structural characteristics of tumor angiogenesis in lung cancers overexpressing different VEGF isoforms assessed by DCE- and SSCE-MRI. PLoS One 6: e16062, 2011.

15. Jia ZZ, Geng DY, Liu Y, Chen XR and Zhang J: Microvascular permeability of brain astrocytoma with contrast-enhanced magnetic resonance imaging: Correlation analysis with histopathologic grade. Chin Med J (Engl) 126: 1953-1956, 2013.

16. Jia ZZ, Gu HM, Zhou XJ, Shi JL, Li MD, Zhou GF and Wu XH: The assessment of immature microvascular density in brain gliomas with dynamic contrast-enhanced magnetic resonance imaging. Eur J Radiol 84: 1805-1809, 2015.

17. Takada K, Yamada S and Teramoto A: Correlation between tumor vascularity and clinical findings in patients with pituitary adenomas. Endocr Pathol 15: 131-139, 2004.

18. Lloyd RV, Scheithauer BW, Kuroki T, Vidal S, Kovacs K and Stefaneanu L: Vascular endothelial growth factor (VEGF) expression in human pituitary adenomas and carcinomas. Endocr Pathol 10: 229-235, 1999.

19. Tofts PS: Modeling tracer kinetics in dynamic Gd-DTPA MR imaging. J Magn Reson Imaging 7: 91-101, 1997.

20. Ruimin L, Yajia G, Jian M, et al: Research of benign and malignant breast lesions differentiation using quantitative DCE-MRI. Chin J Radiol 45: 164-169, 2011 (In Chinese).

21. Wang Y, Huang W, Panicek DM, Schwartz LH and Koutcher JA Feasibility of using limited population-based arterial input function for pharmacokinetic modeling of osteosarcoma dynamic contrast-enhanced MRI data. Magn Reson Med 59: 1183-1189, 2008.

22. Yankeelov TE, Cmn GO, Addison CL, Wallace JC, Wilkins RC, Pappas BA, Santyr GE and Gore JC: Comparison of a reference region model with direct measurement of an AIF in the analysis of DCE-MRI data. Magn Reson Med 57: 353-361, 2007.

23. Vidal S, Kovacs K, Horvath E, Scheithauer BW, Kuroki T and Lloyd RV: Microvessel density in pituitary adenomas and carcinomas. Virchows Arch 438: 595-602, 2001.
24. Turner HE, Nagy Z, Gatter KC, Esiri MM, Harris AL and Wass JA: Angiogenesis in pituitary adenomas-relationship to endocrine function, treatment and outcome. J Endocrinol 165: 475-481, 2000

25. Onguru O, Casey MB, Kajita S, Nakamura N and Lloyd RV: Cyclooxygenase-2 and thromboxane synthase in non-endocrine and endocrine tumors: A review. Endocr Pathol 16: 253-277, 2005.

26. Turner HE, Nagy Z, Gatter KC, Esiri MM, Harris AL and Wass JA: Angiogenesis in pituitary adenomas and the normal pituitary gland. J Clin Endocrinol Metab 85: 1159-1162, 2000.

27. Jugenburg M, Kovacs K, Stefaneanu L and Scheithauer BW: Vasculature in nontumorous hypophyses, pituitary adenomas, and carcinomas: A quantitative morphological study. Endocr Pathol 6: 115-124, 1995.

28. JasekE,Furgal-Borzych A,Lis GJ,Litwin JA,Rzepecka-WozniakE and Trela F: Microvessel density and area in pituitary microadenomas. Endocr Pathol 20: 221-226, 2009.

29. Jeffcoate WJ, Pound N, Sturrock ND and Lambourne J: Long-term follow-up of patients with hyperprolactinaemia. Clin Endocrinol (Oxf) 45: 299-303, 1996.

30. Juneau P, Schoene W C and Black P: Malignant tumors in the pituitary gland. Arch Neurol 49: 555-558, 1992.

31. Lidhar K, Korbonits M, Jordan S, Khalimova Z, Kaltsas G, Lu X, Clayton RN, Jenkins PJ, Monson JP, Besser GM, et al: Low expression of the cell cycle inhibitor p27Kip1 in normal corticotroph cells, corticotroph tumors, and malignant pituitary tumors. J Clin Endocrinol Metab 84: 3823-3830, 1999.

32. Lohrer P, Gloddek J, Hopfner U, Losa M, Uhl E, Pagotto U, Stalla GK and Renner U: Vascular endothelial growth factor production and regulation in rodent and human pituitary tumor cells in vitro. Neuroendocrinology 74: 95-105, 2001.

(i) 9 This work is licensed under a Creative Commons Attribution-NonCommercial-NoDerivatives 4.0 International (CC BY-NC-ND 4.0) License. 